\title{
LINC02418 promotes malignant behaviors in lung adenocarcinoma cells by sponging miR-4677-3p to upregulate KNL1 expression
}

Tao Wang ${ }^{1 \dagger}$, Ruiren Zhai ${ }^{2+}, X_{i u h u a ~} \mathrm{Lv}^{3^{*}}, \mathrm{Ke} \mathrm{Wang}^{3}$ and Junqing $\mathrm{Xu}^{4^{*}}$ (D)

\begin{abstract}
Background: Lung adenocarcinoma (LAD) is a prevalent type of bronchogenic malignant tumor and one of the most critical factors related to human death. Long noncoding RNAs (IncRNAs) are involved in many complex biological processes and have been emerged as extremely important regulators of various cancers. LINC02418, a novel IncRNA, hasn't been mentioned in previous studies on cancer development. Therefore, it's important to define the potential function of LINC02418 in LAD.

Methods: Gene expression was examined by RT-qPCR or western blot. CCK-8, colony formation, TUNEL, and transwell assays were utilized to study the role of LINC02418 in LAD. The interaction of miR-4677-3p with LINC02418 (or KNL1) was verified through luciferase reporter, RIP and RNA pull-down assays.

Results: High expression of LINC02418 was observed in LAD specimens and cells. Downregulation of LINC02418 obstructed the proliferation and motility of LAD cells. Moreover, LINC02418 negatively modulated miR-4677-3p expression and miR-4677-3p overexpression could repress cell proliferation and migration. Moreover, kinetochore scaffold 1 (KNL1) expression was negatively modulated by miR-4677-3p but positively regulated by LINC02418. Furthermore, miR-4677-3p could bind with LINC02418 (or KNL1). Finally, KNL1 overexpression reversed the inhibitory function of LINC02418 deficiency in the malignant behaviors of LAD cells.
\end{abstract}

Conclusions: LINC02418 contributes to the malignancy in LAD via miR-4677-3p/KNL1 signaling, providing a probable therapeutic direction for LAD.

Keywords: LINC02418, miR-4677-3p, KNL1, LAD

\footnotetext{
* Correspondence: 171428256@qq.com; junqingxu@126.com

${ }^{\dagger}$ Tao Wang and Ruiren Zhai are co-first authors.

${ }^{3}$ Department of Radiology, Xijing Hospital, Fourth Military Medical University,

Xi'an 710032, Shaanxi, China

${ }^{4}$ Department of Radiology, Shenzhen University General Hospital, Shenzhen

University Clinical Medical Academy, No.1098 Xueyuan Avenue, Nanshan

District, Shenzhen 518055, Guangdong, China

Full list of author information is available at the end of the article
}

(c) The Author(s). 2020 Open Access This article is licensed under a Creative Commons Attribution 4.0 International License, which permits use, sharing, adaptation, distribution and reproduction in any medium or format, as long as you give appropriate credit to the original author(s) and the source, provide a link to the Creative Commons licence, and indicate if changes were made. The images or other third party material in this article are included in the article's Creative Commons licence, unless indicated otherwise in a credit line to the material. If material is not included in the article's Creative Commons licence and your intended use is not permitted by statutory regulation or exceeds the permitted use, you will need to obtain permission directly from the copyright holder. To view a copy of this licence, visit http://creativecommons.org/licenses/by/4.0/ The Creative Commons Public Domain Dedication waiver (http://creativecommons.org/publicdomain/zero/1.0/) applies to the data made available in this article, unless otherwise stated in a credit line to the data. 


\section{Background}

As a main subtype of non-small cell lung cancer (NSCL $\mathrm{C})$, lung adenocarcinoma (LAD) is one of the leading causes of cancer-related deaths around the world $[1,2]$. Previous studies have identified major pathways involved in LAD development (30-60\%), including the activation of the EGFR, KRAS, and ALK signals [3-5]. Although various molecular targeted therapies have been developed, the prognosis of LAD patients is still disappointing $[6,7]$. Therefore, defining the molecular mechanisms underlying this fatal disease would be of considerable significance for LAD treatment.

Long non-coding RNAs (lncRNAs) are a subtype of non-coding RNAs (ncRNAs), consisting of RNA over 200 nucleotides in length that are not translated into proteins $[8,9]$. Mounting evidence has elucidated a pivotal role of lncRNAs in cancer progression. For example, lncRNA ANCR regulates EZH2 expression to inhibit breast cancer progression [10]. LncRNA LINC00312 expedites cell migration and vasculogenic mimicry in LAD by binding with YBX1 [11]. LncRNA-SNHG1 modulates DNMT1 expression to accelerate the development of gastric cancer [12]. A large number of studies have unmasked that lncRNAs exert critical functions in diverse processes in lung cancer, such as proliferation [13], migration [14] and epithelial-mesenchymal transition (EMT) [15]. Additionally, the involvement of lncRNAs in LAD is also recognized. For instance, lncRNA DGCR5 suppresses the expression of miR-22-3p to promote LAD progression [16]. Galectin-3 activates TLR4/NF- $\mathrm{kB}$ signaling pathway to facilitate the development of LAD via upregulating IncRNA-NEAT1 expression [17]. LncRNA MIR31HG overexpression promotes cell proliferation in LAD and associates with poor prognosis [18]. The oncogenic property of some common lncRNAs in LAD has been widely reported, such as LINC00707 [19], MIR31HG [18], OIP5-AS1 [20], MALAT1 [21], etc. Herein, we intended to probe into the biological role of a novel lncRNA in LAD. Using microarray analysis, differentially expressed lncRNAs were identified. The top ten upregulated IncRNAs in LAD samples were chosen for further analysis in LAD cells. LINC02418 was selected to be the research object in current study.

Mechanistically, lncRNAs can interact with microRNAs (miRNAs) to upregulate messenger RNAs (mRNAs) [22, 23], therefore forming a competing endogenous RNA (ceRNA) pathway. Here, bioinformatics analysis and mechanism-based experiments were used to determine the miRNAs that could bind with LINC02418. Similarly, the target mRNAs of miR4677-3p were identified. In conclusion, this study was designed to investigate whether lncRNA LINC02418 could affect LAD development via regulating its downstream genes.

\section{Methods}

\section{Cell culture and transfection}

Human LAD cell lines (A549, SPC-A1, H1299 and PC9) and normal lung epithelial cells BEAS-2B were bought from the Cell Bank of the Chinese Academy of Sciences and incubated with Dulbecco's modified Eagle's medium (DMEM; Invitrogen Life Technology Inc., Carlsbad, CA), containing $10 \%$ fetal bovine serum (FBS) with $5 \% \mathrm{CO}_{2}$ at $37^{\circ} \mathrm{C}$ in humid air. All cell lines were authenticated via STR profiling before using.

The suppression of LINC02418 expression was achieved by sh-LINC02418\#1/2. Sh-LINC02418\#1 and sh-LINC02418\#2 were obtained from GenePharma (Shanghai, China). LINC02418 and KNL1 were overexpressed with pcDNA3.1 vectors (Invitrogen, Carlsbad, USA). MiR-4677-3p mimics were applied to elevate miR-4677-3p expression. MiR-4677-3p mimics and NC mimics were also bought from GenePharma (Shanghai, China). The transfection of above plasmids was conducted by use of Lipofectamine 2000 agent (Invitrogen).

\section{Clinical samples collection and microarray analysis}

Three pairs of LAD and matched non-cancerous tissue samples were acquired from patients (including two male, one female; two patients $<50$ years old, one patient $>50$ years old; one patient at stage of I-II; two patients at stage of III-IV) who received operation in the Second Affiliated Hospital of Air Force Medical University. Patients enrolled in this study signed the informed consents. Ethic Committee of the Second Affiliated Hospital of Air Force Medical University has approved sample collection of this research. Microarray analysis was implemented to profile the expression of lncRNAs in LAD. In detail, total RNA was isolated from three pairs of tissues and quantified utilizing NanoDrop 2000 (Thermo, Waltham, MA, USA), followed by quality-checking by use of Agilent Bioanalyzer 2100 (Agilent Technologies, Santa Clara, CA, USA). Subsequently, the GeneChip 3'IVT express kit (Affymetrix, Santa Clara, CA, USA) was utilized to label the qualified RNA samples and then Affymetrix GeneChip Primeview Human cDNA microarray was used for hybridization in line with the manufacturer's guides. After that, data were analyzed using GeneChip Scanner 3000 (Affymetrix).

\section{Real-time quantitative polymerase chain reaction (RT- qPCR)}

Total RNA was extracted utilizing TRIzol (Invitrogen; Thermo Fisher Scientific, Inc.) and diluted to $200 \mathrm{ng} / \mathrm{ml}$. Complementary DNA (cDNA) synthesis was conducted via applying Taqman Advanced miRNA cDNA Synthesis Kit or Pyrobest DNA Polymerase and M-MLV Reverse Transcriptase (Thermo Fisher Scientific, USA). RTqPCR was then operated using One Step SYBR ${ }^{\circ}$ Prime 
Script $^{\text {tm }}$ RT-PCR Kit II (Takara Biotechnology Co., Ltd., Dalian, China) based on the producer's protocol. Gene expression relative to GAPDH or U6 was assessed using the $2^{-\Delta \Delta C t}$ method.

\section{Cell counting kit-8 (CCK-8) assay}

Cell proliferation was estimated utilizing the CCK-8 kit (Boster) based on the manufacturer's requirements. Briefly, cells $\left(1 \times 10^{3}\right)$ were supplemented into 96-well plates. After cell adhesion, each well received $10 \mu \mathrm{l}$ CCK-8 solution and then cells were further incubated for $1 \mathrm{~h}$ at $37^{\circ} \mathrm{C}$. Cell proliferation ability was monitored by detecting absorbance at $450 \mathrm{~nm}$ utilizing microplate reader (EL340; Bio-Tek Instruments, Hopkinton, MA, USA) at the indicated time points $(0,24,48,72$ and $96 \mathrm{~h})$.

\section{Colony formation assay}

Cells were seeded in six-well plates and grown in media with 10\% FBS. Two weeks later, the colonies were fixated using methanol and dyed using $0.1 \%$ crystal violet for half an hour. Colonies with over 50 cells were counted manually.

\section{Transwell assay}

For invasion estimation, $5 \times 10^{4}$ cells were added onto the upper chambers (BD Biosciences, San Jose, CA, USA) with Matrigel-coating and incubated in DMEM. DMEM supplementing with $10 \%$ FBS was put into the bottom chambers. Twenty-four hours later, cotton swabs were used to scrape off cells in the upper chamber. The methanol and $0.5 \%$ crystal violet were separately use to fasten and color the cells in the lower chamber. For the migration assays, transfected cells were seeded into the upper chambers with no Matrigel-coating, while other steps were similar to that in invasion assays. Finally, the invaded or migrated cells were counted using an inverted biological microscope (magnification, $\AA \sim 200$ ).

\section{Western blot}

Cells were lysed by use of RIPA (Beyotime, Shangahi, China) containing protease inhibitor cocktail (Roche, Pleasanton, CA) and phenylmethylsulfonyl fluoride (Roche). Protein samples were then subjected to sodium dodecyl sulfate polyacrylamide gel electrophoresis (SDSPAGE), followed by transferring onto nitrocellulose (NC) membranes (Sigma-Aldrich). After blocking via $5 \%$-skim milk, the membranes were cultured with primary antibodies (dilution 1:1000) against Bax, Bcl-2, E-cadherin, N-cadherin, MRP2, MRP9, KNL1 (Cell Signaling Technology, Danvers, MA), followed by incubation with secondary antibodies (dilution 1:10000) for an hour at room temperature. $\beta$-actin or GAPDH was the loading control. Thereafter, signals were captured with the employment of the ECL chromogenic substrate.

\section{Terminal-deoxynucleoitidyl transferase mediated nick end labeling (TUNEL) assay}

After fixation and permeabilization, cells were processed with dUTP-end labeling (Clontech, Mountain View, CA) and (4',6-diamidino-2-phenylindole) DAPI, in succession. After that, cells were observed and analyzed under fluorescent microscope (Olympus, Tokyo, Japan).

\section{Luciferase reporter assay}

A549 and SPC-A1 cells $\left(2.0 \times 10^{4}\right)$ grown in a 96-well plates were co-transfected with $150 \mathrm{ng}$ of LINC02418WT or LINC02418-Mut reporters (Sangon Biotech, Shanghai, China) and miR-4677-3p mimic or NC mimics into LAD cells by use of Lipofectamine 2000 (Invitrogen, Carlsbad, California, USA). KNL1-WT or KNL1-Mut reporters (Sangon Biotech, Shanghai, China) and miR4677-3p mimics or NC mimics were also co-transfected into indicated LAD cells. After transfection for 2 days, the luciferase activity normalized to Renilla luciferase activity was examined by luciferase reporter assay system (Promega, Madison WI).

\section{RNA immunoprecipitation (RIP) assay}

RIP assay was conducted utilizing the EZ-Magna RIP kit (Millipore, Billerica, MA) according to the manufacturer's protocol. A549 and SPCA1 cells at $80-90 \%$ confluence were obtained, and then lysed in complete RIP lysis buffer. Cell extract was processed at $4{ }^{\circ} \mathrm{C}$ for about $6 \mathrm{~h}$ with RIP buffer which contained human Ago2 antibody or control IgG (Millipore) coated magnetic beads. After beads washed, the RNA complexes were cultured with Proteinase $\mathrm{K}$ to digest proteins. RNA concentration was measured though employing a NanoDrop spectrophotometer (Thermo Scientific), with the quality assessed by a bioanalyser (Agilent, Santa Clara, CA). Finally, the immunoprecipitated RNAs were purified and analysed by RT-qPCR.

\section{RNA pull-down assay}

RNA pull-down assay was used to detect the probable interaction among miR-4677-3p, LINC02418 and KNL1. MiR-4677-3p was biotinylated to be miR-4677-3p biotin probe by GenePharma Company (Shanghai, China). MiR-4677-3p biotin probe and miR-4677-3p no-biotin probe were added into the lysates of A549 and SPC-A1 cells. After $48 \mathrm{~h}$ incubation, Dynabeads M-280 Streptavidin (Invitrogen, CA) were put into above mixture. Two hours later, RNA in the pulled down complexes was examined using RT-qPCR analysis after purification. 


\section{Statistical analysis}

Each assay was implemented in triplicate and data were exhibited as mean \pm standard deviation (SD). Based on SPSS for Windows, Version 14.0. (SPSS Inc., Chicago), student's t-test was employed to compare differences between two groups, while one-way analysis of variance (ANOVA) was applied for the comparisons among no less than two groups. $P<0.05$ was deemed as statistically significant.

\section{Results}

\section{The biological function of LINC02418 in LAD}

To identify the molecular mechanisms underlying LAD development, we first examined the differentially expressed lncRNAs in LAD samples relative to paired non-tumor ones. The upregulated lncRNAs in LAD tissues in comparison with adjacent normal tissues were displayed using a heatmap (Fig. 1a). Then, the top ten upregulated lncRNAs in LAD tissues were further analyzed in LAD cell lines via qRT-PCR. Results manifested that compared to the normal BEAS-2B cells, LINC02418 was expressed higher in four LAD cell lines (Fig. 1b; "P<0.01). However, other 9 lncRNAs were not significantly differential expressed in the four kinds of LAD cells relative to control cells (Figure S1A; "P<0.05, " $P<0.01$, n.s. was no significance). Thus, we chose LINC02418 as the research object in subsequent experiments. A549 and SPC-A1 cells possessing highest LINC02418 level were used for loss-of function assays. To probe the biological role of LINC02418, LINC02418 was knocked down in A549 and SPC-A1 cells by transfection with shLINC02418\#1/2, with sh-NC-transfected cells as the scramble control. The results showed that knockdown of LINC02418 resulted in an obvious decline in LINC02418 expression compared with control group

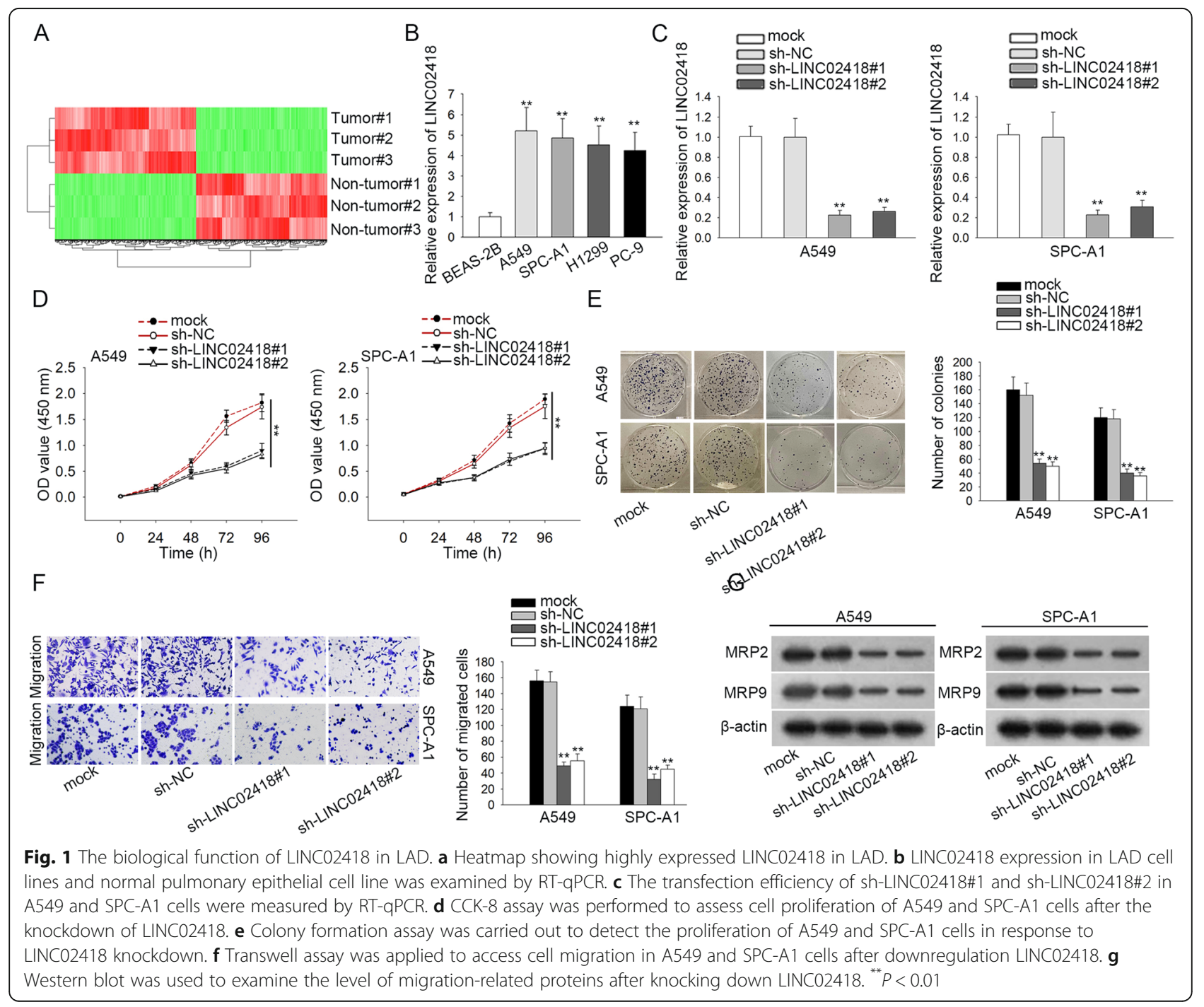


(Fig. 1c; " $P<0.05)$. Additionally, CCK-8 and colony formation assays indicated that LINC02418 absence inhibited the proliferation of these two LAD cells (Fig. 1d-e; ${ }^{* * *} P<0.01$ ). TUNEL assay illustrated that cell apoptosis was promoted by LINC02418 depletion (Figure S2A; $\left.{ }^{* *} P<0.01\right)$. As shown in Fig. If $\left({ }^{* * *} P<0.01\right)$ and S2B $(" P<0.01)$, the migration and invasion of shLINC02418\#1/2-transfected cells were notably blocked in contrast to that in sh-NC group, suggesting that LINC02418 silencing weakened LAD cell migration and invasion abilities. Furthermore, western blot analyses displayed that silencing LINC02418 suppressed the expression of migration-related proteins (MRP2 and MRP9), which indicated that LINC02418 depletion could inhibit cell migration (Fig. 1g). Further, when downregulating LINC02418, the levels of Bax and Ecadherin were augmented while $\mathrm{Bcl}-2$ and $\mathrm{N}$-cadherin expression was declined (Figure S2C). In total, LINC02418 is upregulated and LINC02418 knockdown suppresses cell proliferation and motility in LAD.

\section{The specific role of miR-4677-3p in LAD}

Thereafter, bioinformatics analyses were employed to predict the miRNAs that could possibly bind with LINC02418. As a result, miR-4677-3p was then identified from starBase (Fig. 2a). In order to investigate whether LINC02418 could regulate miR-4677-3p expression, RT-qPCR analysis was performed. Interestingly, an observable increase in the expression of miR-4677-3p was observed in LAD cells with LINC02418 deficiency (Fig. 2b; $\quad P<0.01$ ). Additionally, miR-4677-3p was found to be with a low expression trend in LAD cell lines relative to BEAS-2B cells (Fig. 2 c; ${ }^{\prime \prime \prime} P<0.01$ ). Subsequently, we observed a significantly heightened level of miR-4677-3p in miR-4677-3p mimics group compared with NC mimics group (Fig. 2 d; $" P<0.01$ ). Moreover, miR-4677-3p mimics suppressed cell proliferation in two LAD cells (Fig. 2e-f; ${ }^{\prime \prime} P<0.01$ ). Furthermore, overexpression of miR-4677-3p notably impaired LAD cell migration capability (Fig. 2g; $" P<0.01$ ). Moreover, as displayed in Fig. 2h, miR-4677-3p upregulation

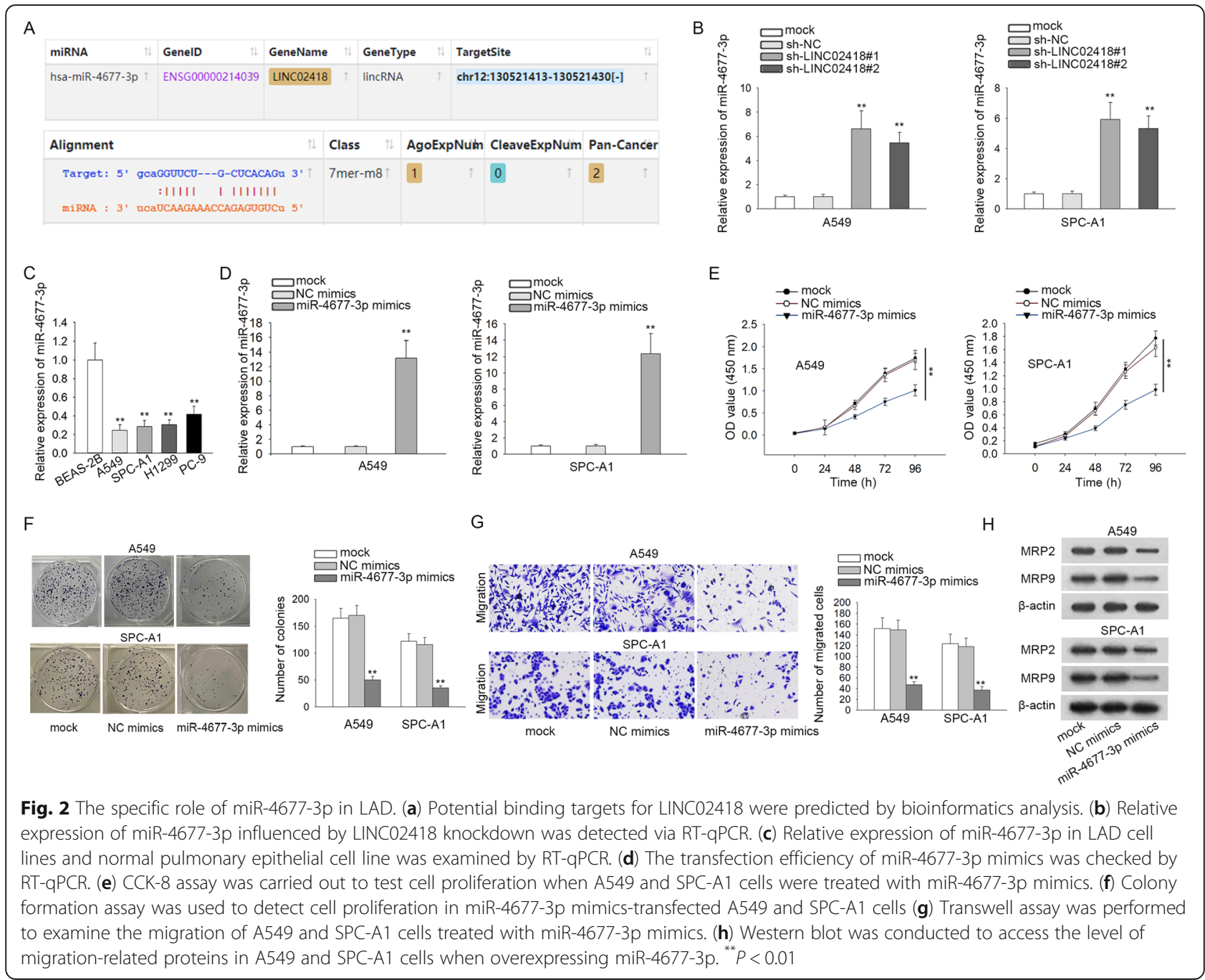


decreased the expression of migration-related proteins (MRP2 and MRP9). Taken together, miR-4677-3p is downregulated and serves a tumor-restraining part in LAD.

\section{KNL1 is a downstream target gene of miR-4677-3p}

Based on research over the past decades, miRNAs are known to have a vital impact on cancer progression by directly modulating the expression of its target genes [24-26]. Herein, 8 candidates (MAP 1LC3B, IGSF3, KNL1, MKL2, EFTUD2, CA12, MOCS1 and SLC31A1) were identified as the potential targets of miR-4677-3p following analyses of the RNA22 and miRmap databases
(Fig. 3a). RT-qPCR was then employed to assess the influence of miR-4677-3p on the expression of these genes in A549 and SPC-A1 cells. Results indicated that only KNL1 expression was notably reduced by miR-4677-3p mimics compared with that in $\mathrm{NC}$ mimics control group in both the two LAD cells, while the level of other genes not (Fig. 3b; " $P<0.05, "{ }^{*} P<0.01$ ). Therefore, KNL1 was selected to carry out the following assays. As illustrated in Fig. 3c, the level of KNL1 protein was also decreased by miR-4677-3p mimics. Besides, both mRNA and protein expressions of KNL1 were also hampered in face of LINC02418 knockdown (Fig. 3d; $" P<0.01$ ). In addition, LINC02418, miR-4677-3p and KNL1 were all

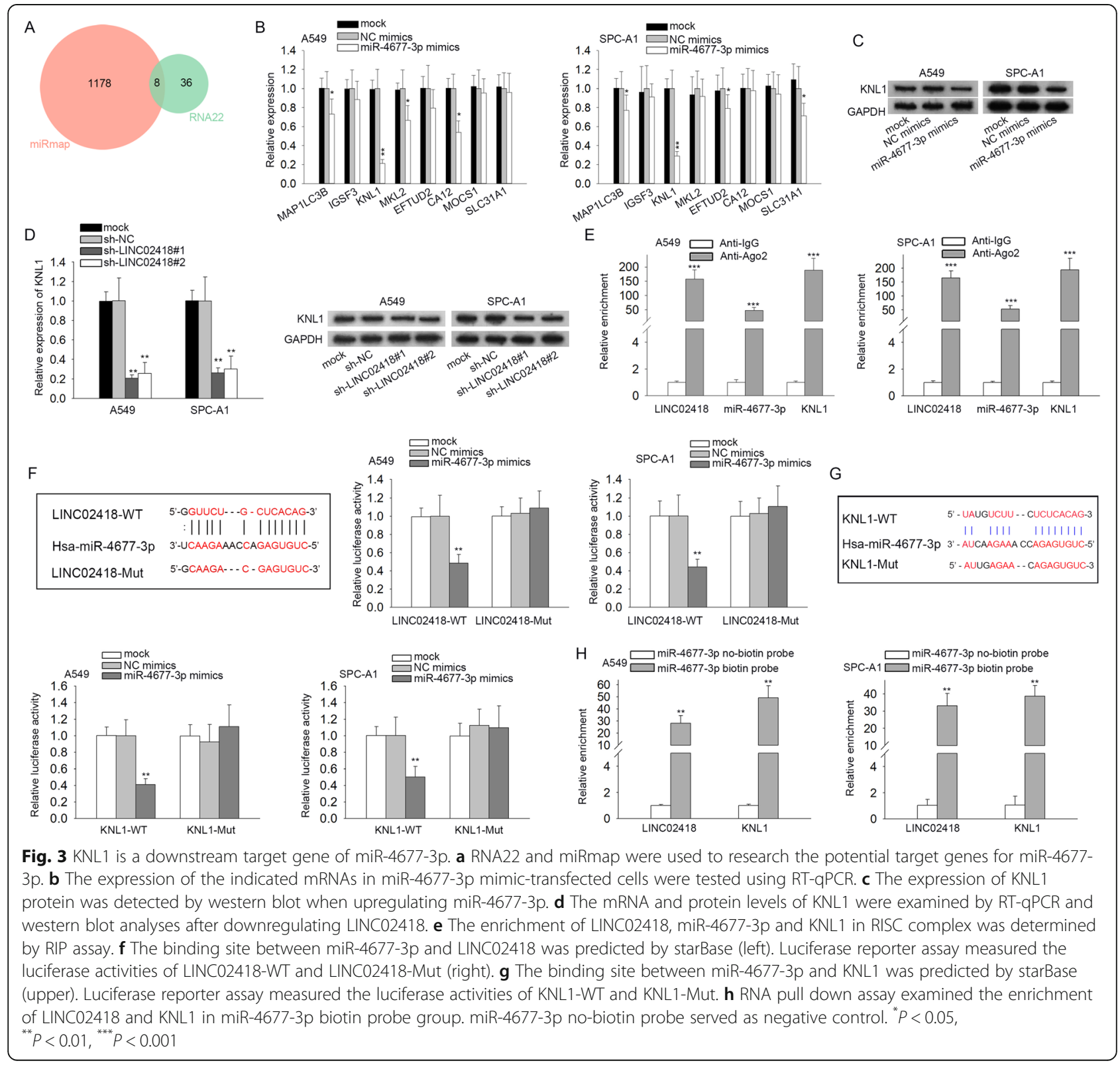


concentrated in anti-Ago2 group instead of anti-IgG group (Fig. 3e; ${ }^{n=0} P<0.001$ ). Furthermore, the binding sequences between miR-4677-3p and LINC02418 were obtained from starBase (Fig. 3f, left). Then we found that the luciferase activity of pmirGLO-LINC02418-WT was reduced by miR-4677-3p mimics whereas that of pmirGLO-LINC02418-Mut showed no obvious alteration between miR-4677-3p mimic group and NC mimic group (Fig. 3f, right; ${ }^{\prime \prime \prime} P<0.01$ ). Similarly, starBase predicted the sequences of miR-4677-3p and KNL1 where the binding occurred (Fig. 3g, upper). The luciferase activity of pmirGLO-KNL1-WT was also lowered by enhanced miR-4677-3p while that of pmirGLO-KNL1-Mut wasn't affected (Fig. 3g, lower; ${ }^{* * \prime} P<0.01$ ). Moreover, the results of RNA pull-down assay testified that both LINC02418 and KNL1 were enriched in miR-4677-3p biotin probe group (Fig. $3 \mathrm{~h} ;{ }^{* *} P<0.01$ ). In sum, KNL1 is the downstream molecule of miR-4677-3p in LAD.

\section{LINC02418 promotes the malignant phenotypes of LAD cells through miR-4677-3p/KNL1 signaling}

Subsequently, we planned to determine whether LINC02418 influenced LAD development through miR-4677-3p/KNL1 pathway. RT-qPCR and western blot analyses indicated that co-transfection of pcDNA3.1/KNL1 recovered LINC02418 depletionlessened KNL1 expression in A549 cells (Fig. 4a-b; " $P<0.01$, n.s. was no significance). Then, it was verified that KNL1 upregulation reversed the hindering impact of LINC02418 deficiency on A549 cell proliferation (Fig. 4c-d; ${ }^{* \prime \prime} P<0.01$, n.s. was no significance). Additionally, the stimulated cell apoptosis induced by LINC02418 silencing was rescued by KNL1 overexpression (Figure S3A; $P<0.01$, n.s. was no significance). Subsequently, it was confirmed that overexpression of KNL1 offset the obstructive function caused by LINC02418 depletion on A549 cell motility (Fig. 4e and S3B; $"$ P $P<0.01$, n.s. was no significance). Additionally, KNL1 upregulation neutralized the influence of LINC02418 depletion on the levels of Bax, Bcl-2, Ecadherin and N-cadherin (Figure S3C). These findings suggest that LINC02418 aggravates malignant behaviors in LAD via miR-4677-3p/KNL1 pathway.

\section{Discussion}

Lung adenocarcinoma (LAD) is the most common subtype of non-small-cell lung cancer (NSCLC), accounting for the majority of diagnosed primary lung cancer cases, and also with low 5-year survival rate $[27,28]$. In recent few decades, great progresses have been achieved in LAD treatment, including anti-PD-1/ PD-L1 therapy and targeted therapy [29, 30]. In the meantime, treatment strategies for LAD have also improved [31-33]. Nonetheless, the survival rate of patients with LAD still remains poor. Hence, identifying effective targets is imperative for opening fresh strategies for LAD treatment.

Recently, a great amount of work has uncovered the veil of lncRNAs in multiple cancers, and a growing body of literature has delineated that lncRNAs are implicated in various malignancies, such as LAD, breast cancer and gastric cancer [12, 34, 35]. Nonetheless, whether LINC02418 works in LAD has not been revealed. Currently, we found the notable upregulation of LINC02418 in LAD, and the absence of LINC02418 suppressed LAD cell proliferation and motility, implying that LINC02418 promotes the malignancy in LAD.

MiRNAs are also defined as a fraction of ncRNAs with the length of 20-24 nucleotides [36, 37]. Previous research suggests that miRNAs exert their functions in diverse cancers [38, 39]. As an illustration, miR-4500 is downregulated and elicits an anti-cancer function through regulating HMGA2 expression in colorectal cancer [40]. MiR-431-5p targets UROC28 to influence the expression of EMT markers in hepatocellular carcinoma [41]. MiR-205 regulates E2F1 expression to promote the cisplatin sensitivity of glioma cells [42]. In LAD, miRNAs also play important roles, like miR-133 participates in LAD metastasis via targeting with FLOT2 [43]. MiR-629-3p inhibits SFTPC expression to facilitate cell proliferation and is associated with poor survival in LAD [44]. MiR-608 and miR-4513 greatly enhance the prognosis of LAD treated with EGFR-TKIs [45]. Present study showed the decreased expression of miR-4677-3p in LAD and revealed that miR-4677-3p overexpression suppressed cell proliferation and migration in LAD, highlighting miR-4677-3p as a cancer-suppressor in LAD.

Proteins translated from messenger RNAs (mRNAs) play critical roles in cancer. For instance, EGFR enhances the development of renal cancer [46]. YWHAZ serves as an oncogenic gene in cervical cancer [47]. Also, the oncogenic function of KNL1 has been validated in cancer. For example, miR-193b-3p silencing promotes cell proliferation in gastric cancer through upregulating the expression of KNL1 [48]. CeRNA hypothesis have been proposed and proven to be transcripts cross-regulated by competing certain miRNAs $[49,50]$. To be specific, lncRNA and mRNA can competitively bind with the shared miRNA to modulate cancer progression. For instance, IncRNA HOXD-AS1 promotes liver cancer metastasis through sponging miR-130a-3p and targeting SOX4 [51]. LncRNA TUG1 facilitates the development of papillary thyroid cancer via miR-145/ZEB1 axis [52]. LncRNA-UCA1 exerts its oncogenic function in esophageal cancer through acting as the ceRNA of 


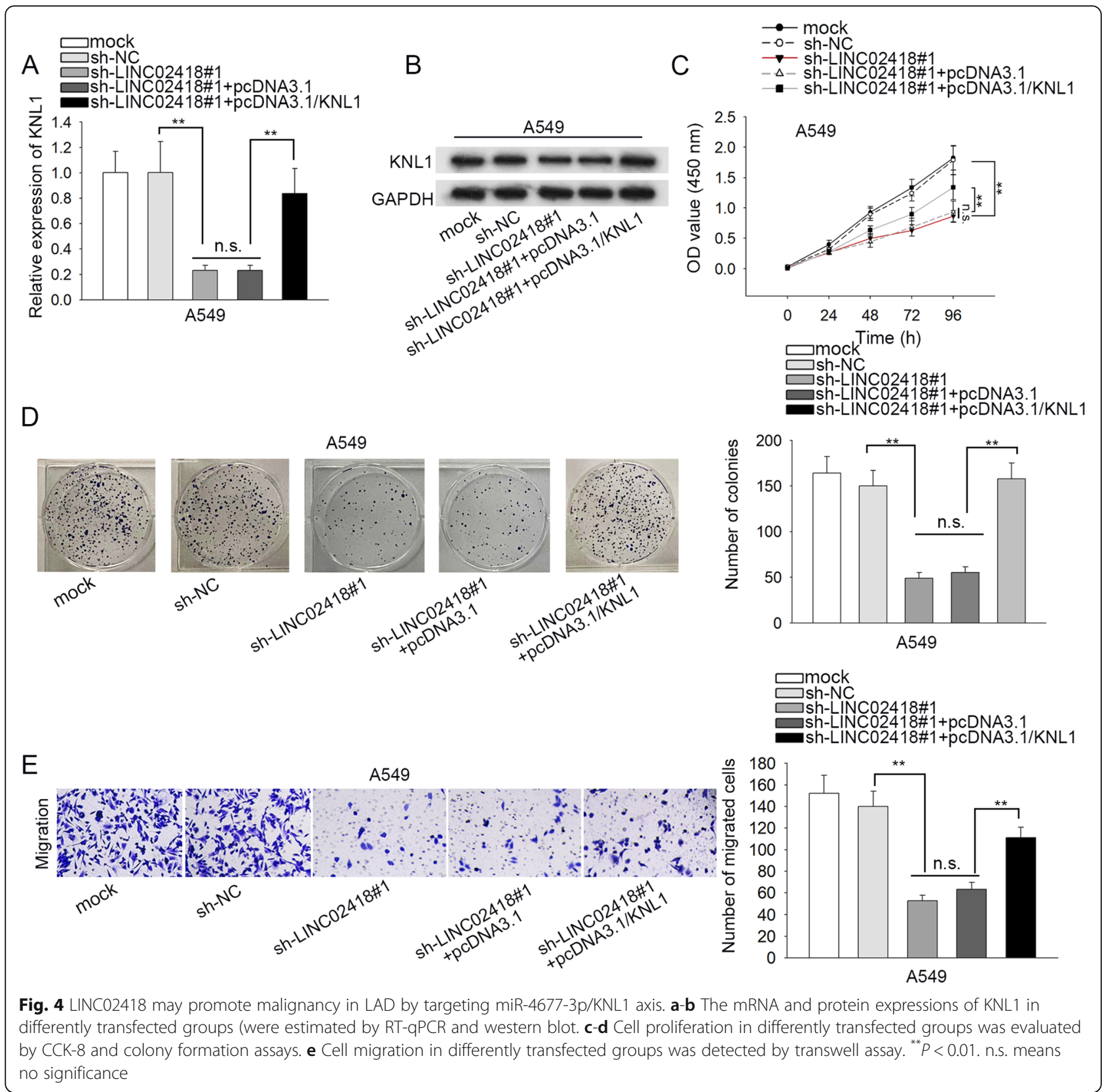

SOX4 [53]. Our study revealed that miR-4677-3p could bind with LINC02418 and KNL1, and KNL1 was the mediator downstream of LINC02418/miR4677-3p signaling in LAD. Finally, rescue assays indicated that the inhibited LAD cell functions induced by LINC02418 silencing were counteracted by KNL1 overexpression.

Upregulation of LINC02418 in LAD tissue samples was identified by a microarray analysis, indicating the clinical potential of LINC02418 in LAD patients. This study didn't elucidate the role of LINC02418 in clinical features or prognosis. Thus, we will investigate the clinical value of LINC02418 in LAD in future research. In conclusion, LINC02418 contributes to malignant phenotypes of LAD cells through sequestering miR-4677-3p to boost KNL1 level, throwing light on the molecular mechanism of LINC02418 in LAD, providing a novel target for LAD treatment.

\section{Conclusions}

LINC02418 facilitates malignant cell behaviors in LAD via sponging miR-4677-3p to upregulate KNL1 expression. 


\section{Supplementary information}

Supplementary information accompanies this paper at https://doi.org/10. 1186/s12890-020-01229-0.

Additional file 1: Figure S1. (A) Expression pattern of 9 IncRNAs in LAD cells and normal BEAS-2B cell was tested by RT-qPCR. ${ }^{*} P<0.05,{ }^{* *} P<$ 0.01 ; n.s.: no significance.

Additional file 2: Figure S2. (A) TUNEL assay measured cell apoptosis in LINC02418 downregulated cells. (B) Transwell assay detected cell invasion when knocking down LINC02418. (C) Western blot tested expression of apoptosis- and EMT-related proteins in response to LINC02418 depletion. ${ }^{* *} P<0.01$

Additional file 3: Figure S3. (A) TUNEL assay measured cell apoptosis in differently transfected groups. (B) Transwell assay detected cell invasion in differently transfected groups. (C) Western blot tested expression of apoptosis- and EMT-related proteins in differently transfected groups. ${ }^{* *} \mathrm{P}<0.01$; n.s.: no significance.

Additional file 4: Supplementary Information file 1. The original, unprocessed gel images for western blot data in Figs. 1g, 2h, 3c, d, 4b, S2C and S3C.

\section{Abbreviations}

ANCR: Angelman syndrome chromosome region; ANOVA: Analysis of variance; CA12: Carbonic anhydrase 12; CCK-8: Cell counting kit-8; ceRNA: Competing endogenous RNA; DGCR5: DiGeorge syndrome critical region gene 5; DMEM: Dulbecco's modified Eagle's medium; DNMT1: DNA methyltransferase 1; E2F1: E2F transcription factor 1; EFTUD2: Elongation factor Tu GTP binding domain containing 2; EGFR: Epidermal growth factor receptor; EMT: Epithelial-mesenchymal transition; EZH2: Enhancer of zeste 2 polycomb repressive complex 2 subunit; FBS: Fetal bovine serum; GAS5: Growth arrest specific 5; HMGA2: High mobility group AT-hook 2 HOTAIR: HOX transcript antisense RNA; HOXD-AS1: HOXD antisense growthassociated long non-coding RNA; IGSF3: Immunoglobulin superfamily member 3; KNL1: Kinetochore scaffold 1; LAD: Lung adenocarcinoma; LINC02418: Long intergenic non-protein coding RNA 2418; IncRNAs: Long noncoding RNAs; MALAT1: Metastasis associated lung adenocarcinoma transcript 1; MAP 1 LC3B: Microtubule associated protein 1 light chain 3 beta; MIR31HG: MIR31 host gene; miRNAs: microRNAs; MKL2: Myocardin like 2; MOCS1: Molybdenum cofactor synthesis 1; n.s.: no significance; NEAT1: Nuclear paraspeckle assembly transcript 1; NSCLC: Non-small cell lung cancer; PVT1: Pvt1 oncogene; RIP: RNA immunoprecipitation; RIPA: Radioimmunoprecipitation assay; RT-qPCR: Real-time quantitative polymerase chain reaction; SD: Standard deviation; SLC31A1: Solute carrier family 31 member 1; SNHG1: Small nucleolar RNA host gene 1; SOX4: SRYbox transcription factor 4; TIMP2: TIMP metallopeptidase inhibitor 2; TLR4: Toll like receptor 4; TUG1: Taurine up-regulated 1; TUNEL: Terminaldeoxynucleoitidyl transferase mediated nick end labeling; UCA1: Urothelial cancer associated 1; YWHAZ: Tyrosine 3-monooxygenase/tryptophan 5monooxygenase activation protein zeta; ZEB1: Zinc finger E-box binding homeobox 1

\section{Acknowledgements}

We appreciate all the people involved in this study.

\section{Authors' contributions}

TW and RZ: conceptualization, project administration, manuscript review, data analysis; $\mathrm{XL}$ and $\mathrm{KW}$ : investigation, experiment record, figures; JX: correspondence, original manuscript writing. All authors gave useful advices. The author(s) read and approved the final manuscript002E

\section{Funding}

None.

\section{Availability of data and materials}

Relevant data and materials have been presented within the manuscript and additional files.

\section{Ethics approval and consent to participate}

This study was approved by the Ethics Committee of the Second Affiliated Hospital of Air Force Medical University. Patients involved in this work signed the informed consents before sample collection.

\section{Consent for publication}

Not applicable.

\section{Competing interests}

The authors declare that they have no competing interests.

\section{Author details}

${ }^{1}$ Department of Thoracic Surgery, The Second Affiliated Hospital of Air Force Medical University, Xi'an 710038, Shaanxi, China. ${ }^{2}$ Department of Tumor Center, Sunshine Union Hospital, Weifang 261000, Shandong, China. ${ }^{3}$ Department of Radiology, Xijing Hospital, Fourth Military Medical University, Xi'an 710032, Shaanxi, China. ${ }^{4}$ Department of Radiology, Shenzhen University General Hospital, Shenzhen University Clinical Medical Academy, No.1098 Xueyuan Avenue, Nanshan District, Shenzhen 518055, Guangdong, China.

Received: 6 June 2019 Accepted: 8 July 2020

Published online: 14 August 2020

\section{References}

1. Chou J, Wang B, Zheng T, Li X, Zheng L, Hu J, Zhang Y, Xing Y, Xi T. MALA $\mathrm{T} 1$ induced migration and invasion of human breast cancer cells by competitively binding miR-1 with cdc42. Biochem Biophys Res Commun. 2016:472(1):262-9.

2. Wei X, Zhang K, Qin H, Zhu J, Qin Q, Yu Y, Wang H. GMDS knockdown impairs cell proliferation and survival in human lung adenocarcinoma. BMC Cancer. 2018;18(1):600

3. Janku F, Stewart DJ, Kurzrock R. Targeted therapy in non-small-cell lung cancer--is it becoming a reality? Nat Rev Clin Oncol. 2010;7(7):401-14.

4. Ihle NT, Byers LA, Kim ES, Saintigny P, Lee JJ, Blumenschein GR, Tsao A, Liu S, Larsen JE, Wang J, et al. Effect of KRAS oncogene substitutions on protein behavior: implications for signaling and clinical outcome. J Natl Cancer Inst. 2012;104(3):228-39.

5. Pao W, Girard N. New driver mutations in non-small-cell lung cancer. Lancet Oncol. 2011;12(2):175-80

6. Morgensztern D, Campo MJ, Dahlberg SE, Doebele RC, Garon E, Gerber DE, Goldberg SB, Hammerman PS, Heist RS, Hensing T, et al. Molecularly targeted therapies in non-small-cell lung cancer annual update 2014. J Thorac Oncol. 2015:10(1 Suppl 1):S1-63.

7. Siegel RL, Miller KD, Jemal A. Cancer statistics, 2016. CA Cancer J Clin. 2016; 66(1):7-30.

8. Zheng Y, Liu L, Shukla GC. A comprehensive review of web-based noncoding RNA resources for cancer research. Cancer Lett. 2017:407:1-8.

9. Gomes CC, de Sousa SF, Calin GA, Gomez RS. The emerging role of long noncoding RNAs in oral cancer. Oral Surg Oral Med Oral Pathol Oral Radiol. 2017;123(2):235-41.

10. Zhao L, Wu D, Sang M, Xu Y, Liu Z, Wu Q. Stachydrine ameliorates isoproterenol-induced cardiac hypertrophy and fibrosis by suppressing inflammation and oxidative stress through inhibiting NF-kappaB and JAK STAT signaling pathways in rats. Int Immunopharmacol. 2017:48:102-9.

11. Peng Z, Wang J, Shan B, Li B, Peng W, Dong Y, Shi W, Zhao W, He D, Duan $\mathrm{M}$, et al. The long noncoding RNA LINC00312 induces lung adenocarcinoma migration and vasculogenic mimicry through directly binding YBX1. Mol Cancer. 2018:17(1):167.

12. Hu Y, Ma Z, He Y, Liu W, Su Y, Tang Z. LncRNA-SNHG1 contributes to gastric cancer cell proliferation by regulating DNMT1. Biochem Biophys Res Commun. 2017:491(4):926-31.

13. Jing $H$, Qu X, Liu L, Xia H. A Novel Long Noncoding RNA (IncRNA), LL22NC03-N64E9.1, Promotes the Proliferation of Lung Cancer Cells and is a Potential Prognostic Molecular Biomarker for Lung Cancer. Med Sci Monit. 2018:24:4317-23.

14. Hao Y, Yang X, Zhang D, Luo J, Chen R. Long noncoding RNA LINC01186, regulated by TGF-beta/SMAD3, inhibits migration and invasion through epithelial-Mesenchymal-transition in lung cancer. Gene. 2017;608:1-12.

15. Pan C, Yao G, Liu B, Ma T, Xia Y, Wei K, Wang J, Xu J, Chen L, Chen Y. Long noncoding RNA FAL1 promotes cell proliferation, invasion and epithelial- 
Mesenchymal transition through the PTEN/AKT signaling Axis in non-smal cell lung Cancer. Cell Physiol Biochem. 2017;43(1):339-52

16. Dong HX, Wang R, Jin XY, Zeng J, Pan J. LncRNA DGCR5 promotes lung adenocarcinoma (LUAD) progression via inhibiting hsa-mir-22-3p. J Cell Physiol. 2018;233(5):4126-36.

17. Zhou W, Chen X, Hu Q, Chen X, Chen Y, Huang L. Galectin-3 activates TLR4/ NF-kappaB signaling to promote lung adenocarcinoma cell proliferation through activating InCRNA-NEAT1 expression. BMC Cancer. 2018;18(1):580

18. Qin J, Ning H, Zhou Y, Hu Y, Yang L, Huang R. LncRNA MIR31HG overexpression serves as poor prognostic biomarker and promotes cells proliferation in lung adenocarcinoma. Biomed Pharmacother. 2018;99:363-8.

19. Ma T, Ma H, Zou Z, He X, Liu Y, Shuai Y, Xie M, Zhang Z. The long Intergenic noncoding RNA 00707 promotes lung adenocarcinoma cell proliferation and migration by regulating Cdc42. Cell Physiol Biochem. 2018;45(4):1566-80

20. Deng J, Deng H, Liu C, Liang Y, Wang S. Long non-coding RNA OIP5-AS1 functions as an oncogene in lung adenocarcinoma through targeting miR448/BCl-2. Biomed Pharmacother. 2018:98:102-10.

21. Li S, Ma F, Jiang K, Shan H, Shi M, Chen B. Long non-coding RNA metastasis-associated lung adenocarcinoma transcript 1 promotes lung adenocarcinoma by directly interacting with specificity protein 1 . Cancer Sci. 2018:109(5):1346-56.

22. Li XY, Zhou LY, Luo H, Zhu Q, Zuo L, Liu GY, Feng C, Zhao JY, Zhang YY, Li $X$. The long noncoding RNA MIR210HG promotes tumor metastasis by acting as a ceRNA of miR-1226-3p to regulate mucin-1c expression in invasive breast cancer. Aging. 2019;11(15):5646-65.

23. Wang L, Luan T, Zhou S, Lin J, Yang Y, Liu W, Tong X, Jiang W. LncRNA HCP5 promotes triple negative breast cancer progression as a ceRNA to regulate BIRC3 by sponging miR-219a-5p. Cancer Med. 2019;8(9):4389-403.

24. Wang W, Yuan X, Xu A, Zhu X, Zhan Y, Wang S, Liu M. Human cancer cells suppress behaviors of endothelial progenitor cells through miR-21 targeting IL6R. Microvasc Res. 2018;120:21-8.

25. Jin K, Zhao W, Xie X, Pan Y, Wang K, Zhang H. MiR-520b restrains cell growth by targeting HDAC4 in lung cancer. Thoracic cancer. 2018;9(10): 1249-54.

26. Chen Z, Hu X, Wu Y, Cong L, He X, Lu J, Feng J, Liu D. Long non-coding RNA XIST promotes the development of esophageal cancer by sponging miR-494 to regulate CDK6 expression. Biomed Pharmacother. 2019;109:2228-36.

27. Kadara $\mathrm{H}$, Kabbout $\mathrm{M}$, Wistuba II. Pulmonary adenocarcinoma: a renewed entity in 2011. Respirology (Carlton). 2012;17(1):50-65.

28. Stahel RA. Adenocarcinoma, a molecular perspective. Ann Oncol. 2007; 18(Suppl 9):ix147-9.

29. Teglasi V, Reiniger L, Fabian K, Pipek O, Csala I, Bago AG, Varallyai P, Vizkelet L, Rojko L, Timar J, et al. Evaluating the significance of density, localization, and PD-1/PD-L1 immunopositivity of mononuclear cells in the clinical course of lung adenocarcinoma patients with brain metastasis. Neurooncology. 2017;19(8):1058-67.

30. Liu D, Vokes NI, Van Allen EM. Toward molecularly driven precision medicine in lung adenocarcinoma. Cancer Discov. 2017;7(6):555-7.

31. Masuda T, Hattori N, Hamada A, Iwamoto H, Ohshimo S, Kanehara M, Ishikawa N, Fujitaka K, Haruta Y, Murai H, et al. Erlotinib efficacy and cerebrospinal fluid concentration in patients with lung adenocarcinoma developing leptomeningeal metastases during gefitinib therapy. Cancer Chemother Pharmacol. 2011:67(6):1465-9.

32. Gottfried M, Bennouna J, Bondarenko I, Douillard JY, Heigener DF, Krzakowski M, Mellemgaard A, Novello S, Orlov S, Summers Y, et al. Efficacy and safety of Nintedanib plus Docetaxel in patients with advanced lung adenocarcinoma: complementary and exploratory analyses of the phase III LUME-lung 1 study. Target Oncol. 2017;12(4):475-85.

33. Bittner N, Ostoros G, Geczi L. New treatment options for lung adenocarcinoma--in view of molecular background. Pathol Oncol Res. 2014; 20(1):11-25.

34. Zhou J, Xiao H, Yang X, Tian H, Xu Z, Zhong Y, Ma L, Zhang W, Qiao G, Liang J. Long noncoding RNA CASC9.5 promotes the proliferation and metastasis of lung adenocarcinoma. Sci Rep. 2018;8(1):37.

35. Li Z, Hou P, Fan D, Dong M, Ma M, Li H, Yao R, Li Y, Wang G, Geng P, et al. The degradation of $\mathrm{EZH} 2$ mediated by IncRNA ANCR attenuated the invasion and metastasis of breast cancer. Cell Death Differ. 2017;24(1):59-71.

36. D'Angelo B, Benedetti E, Cimini A, Giordano A. MicroRNAs: a puzzling tool in Cancer diagnostics and therapy. Anticancer Res. 2016;36(11):5571-5.

37. Cui J, Zhou B, Ross SA, Zempleni J. Nutrition, microRNAs, and Human Health. Adv Nutr (Bethesda). 2017;8(1):105-12.
38. Cheng Y, Ji R, Yue J, Yang J, Liu X, Chen H, Dean DB, Zhang C. MicroRNAs are aberrantly expressed in hypertrophic heart: do they play a role in cardiac hypertrophy? Am J Pathol. 2007;170(6):1831-40.

39. Djuranovic S, Nahvi A, Green R. miRNA-mediated gene silencing by translational repression followed by mRNA deadenylation and decay. Science. 2012;336(6078):237-40.

40. Yu FY, Tu Y, Deng Y, Guo C, Ning J, Zhu Y, Lv X, Ye H. MiR-4500 is epigenetically downregulated in colorectal cancer and functions as a novel tumor suppressor by regulating HMGA2. Cancer Biol Ther. 2016;17(11):1149-57.

41. Kong Q, Han J, Deng H, Wu F, Guo S, Ye Z. miR-431-5p alters the epithelialto-mesenchymal transition markers by targeting UROC28 in hepatoma cells. Onco Targets Ther. 2018;11:6489-503.

42. Li FF, Xing C, Wu LL, Xue F. MiR-205 enhances cisplatin sensitivity of glioma cells by targeting E2F1. Eur Rev Med Pharmacol Sci. 2018;22(2):299-306.

43. Wei G, Xu Y, Peng $T$, Yan J. miR-133 involves in lung adenocarcinoma cell metastasis by targeting FLOT2. Artif Cells Nanomed Biotechnol. 2018;46(2): 224-30.

44. Li B, Meng YQ, Li Z, Yin C, Lin JP, Zhu DJ, Zhang SB. MiR-629-3p-induced downregulation of SFTPC promotes cell proliferation and predicts poor survival in lung adenocarcinoma. Artif Cells Nanomed Biotechnol. 2019; 47(1):3286-96.

45. Zhang N, Li Y, Zheng Y, Zhang L, Pan Y, Yu J, Yang M. miR-608 and miR4513 significantly contribute to the prognosis of lung adenocarcinoma treated with EGFR-TKls. Lab Invest. 2019:99(4):568-76.

46. Wang A, Bao Y, Wu Z, Zhao T, Wang D, Shi J, Liu B, Sun S, Yang F, Wang L, et al. Long noncoding RNA EGFR-AS1 promotes cell growth and metastasis via affecting HuR mediated mRNA stability of EGFR in renal cancer. Cell Death Dis. 2019;10(3):154.

47. Ji N, Wang Y, Bao G, Yan J, Ji S. LncRNA SNHG14 promotes the progression of cervical cancer by regulating miR-206/NWHAZ. Pathol Res Pract. 2019; 215(4):668-75.

48. Song B, Du J, Song DF, Ren JC, Feng Y. Dysregulation of NCAPG, KNL1, miR148a-3p, miR-193b-3p, and miR-1179 may contribute to the progression of gastric cancer. Biol Res. 2018;51(1):44.

49. Qi X, Zhang DH, Wu N, Xiao JH, Wang X, Ma W. ceRNA in cancer: possible functions and clinical implications. J Med Genet. 2015;52(10):710-8.

50. Salmena L, Poliseno L, Tay Y, Kats L, Pandolfi PP. A ceRNA hypothesis: the Rosetta stone of a hidden RNA language? Cell. 2011;146(3):353-8.

51. Wang H, Huo X, Yang XR, He J, Cheng L, Wang N, Deng X, Jin H, Wang N, Wang C, et al. STAT3-mediated upregulation of IncRNA HOXD-AS1 as a ceRNA facilitates liver cancer metastasis by regulating SOX4. Mol Cancer. 2017:16(1):136.

52. Lei H, Gao Y, Xu X. LncRNA TUG1 influences papillary thyroid cancer cell proliferation, migration and EMT formation through targeting miR-145. Acta Biochim Biophys Sin. 2017;49(7):588-97.

53. Jiao C, Song Z, Chen J, Zhong J, Cai W, Tian S, Chen S, Yi Y, Xiao Y. IncRNAUCA1 enhances cell proliferation through functioning as a ceRNA of Sox4 in esophageal cancer. Oncol Rep. 2016:36(5):2960-6.

\section{Publisher's Note}

Springer Nature remains neutral with regard to jurisdictional claims in published maps and institutional affiliations.

Ready to submit your research? Choose BMC and benefit from

- fast, convenient online submission

- thorough peer review by experienced researchers in your field

- rapid publication on acceptance

- support for research data, including large and complex data types

- gold Open Access which fosters wider collaboration and increased citations

- maximum visibility for your research: over $100 \mathrm{M}$ website views per year

At BMC, research is always in progress.

Learn more biomedcentral.com/submissions 